\title{
TEXTURE CONTROL AND THE YIELD ANISOTROPY OF PLANE STRAIN MAGNESIUM EXTRUSIONS
}

\author{
I. L. DILLAMORE†, P. HADDEN $\ddagger$ and D. J. STRATFORD§
}

(Received September 13, 1971)

\begin{abstract}
The textures developed in magnesium, annealed after both hot and cold extrusion under essentially plane strain conditions, have been qualitatively accounted for in terms of the operative deformation modes. It is found that the main features of both textures are explained by the operation of $\{10 \overline{1} 2\}$ and $\{10 \overline{1} 1\}$ twinning together with basal slip, but there is an important component of the hot extrusion annealing texture not found in the cold extrusions texture which can only be explained by the action of $\{10 \overline{10}\}$ or $\{10 \overline{1} 1\}$ slip.

The strength of the extruded and annealed material has been studied under various stress systems and initial yield loci have been plotted. Complete crystal orientation distribution functions were used to compute theoretical yield loci for comparison with the experimental results in order to assess the possibility of relating texture and properties in hexagonal metals. It was found that the theory gave remarkably good agreement with experiment. The assumptions made and the terms under which they apply are discussed.
\end{abstract}

\section{INTRODUCTION}

Metals having the close-packed hexagonal crystal structure are the most prone to exhibit marked anisotropy of physical and mechanical properties. This has been recognised for some time and was well illustrated in the work of Hosford and Backofen ${ }^{1,2}$. Among the hexagonal metals, titanium and zirconium have received the most attention in recent years, the latter in respect of its role in nuclear technology and the former for its promise in chemical engineering and aero-space applications. Perhaps the most under-utilised of the commonly occurring metals is the close-packed hexagonal metal magnesium, this is lighter than all potentially useful engineering metals, with the possible exception of beryllium; it has the ability to submit to forming operations at quite low temperatures, while at room temperature it fails after yield.

One of us ${ }^{3}$ has recently developed a high strength heat-treatable magnesium alloy having a $0.1 \%$ proof stress of up to $370 \mathrm{~N} / \mathrm{mm}^{2}$, which makes its strength to weight ratio superior to that of the best available aluminium alloy. Preliminary experiments ${ }^{4}$ suggest that part of the strengthening is

† Bisra, Corporate Laboratories of BSC, Hoyle Street, Sheffield S3 7EY, England.

\# Department of Physical Metallurgy, University of Birmingham, Edgbaston, Birmingham 15, England.

$\S$ Clayton Dewandre Ltd., No. 3 Factory, Outer Circle Drive, Lincoln, England. crystallographic in origin since different process routes, namely hot or cold extrusion prior to solution treatment, gave marked differences in the as-solution treated strength level, which persisted after precipitation hardening.

It is obvious that to extract the full potential from such materials the crystallographic contribution to strengthening must be much better understood than at present. There is, however, no systematic work on texture control in magnesium and there is very little work on the relationship between texture and properties in magnesium. The present work attempts to fill this gap in part, by studying the textures developed in hot and cold plane strain extrusions of commercial purity magnesium and by further investigating the yielding behaviour under various stress states of the two materials.

\section{EXPERIMENTAL PROCEDURES}

Two billets of commercial purity magnesium, one hot extruded and the other cold extruded, were used in this investigation. The chemical analyses of trace elements are shown in Table I.

Both extrusions were made from a container held at $420^{\circ} \mathrm{C}$, the hot extrusion was from a billet temperature of $350^{\circ} \mathrm{C}$ and for the cold extrusion the billet temperature was $20^{\circ} \mathrm{C}$. In both cases $95.25 \mathrm{~mm}$ diameter billets were extruded to $50.8 \mathrm{~mm}$ by $3.2 \mathrm{~mm}$ rectangular cross-section, a nominal $98.2 \%$ reduction of area, at a product 
TABLE I

\begin{tabular}{lccccccc}
\hline & $\mathrm{Zn}$ & $\mathrm{Al}$ & $\mathrm{Mn}$ & $\mathrm{Cu}$ & $\mathrm{Si}$ & $\mathrm{Fe}$ & $\mathrm{Ni}$ \\
\cline { 2 - 7 } Cold extruded & 0.0055 & 0.0090 & 0.0055 & 0.001 & 0.0055 & 0.010 & $<0.003$ \\
Hot extruded & 0.0060 & 0.0150 & 0.0065 & 0.001 & 0.0025 & 0.005 & $<0.003$ \\
\hline
\end{tabular}

speed of $1.8-2.4 \mathrm{~m} / \mathrm{min}$. Both the hot and cold extrusions were recrystallised as-received but were given a grain growth anneal at $350^{\circ} \mathrm{C}$ to make the grain sizes more nearly equal. The annealing time was 15 minutes for the cold extrusion and 5 minutes for the hot extrusion. There remained some variation in grain size along the length of the extrusions, as shown in Figure 1.

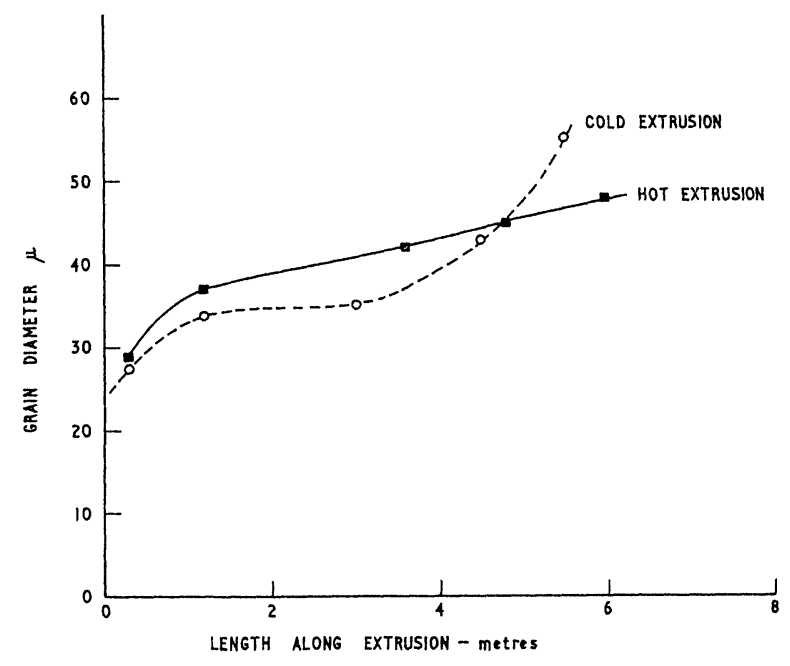

FIGURE 1 Grain size variation along the lengths of the two extrusions, after annealing for 5 minutes and 15 minutes at $350^{\circ} \mathrm{C}$ for the hot and cold extrusions respectively.

Tensile properties along the extrusion and transverse directions were obtained from specimens of overall length $50.8 \mathrm{~mm}$ with $19 \mathrm{~mm}$ gauge length, $6.35 \mathrm{~mm}$ gauge width and $3.2 \mathrm{~mm}$ thickness. Compression tests were carried out parallel to the extrusion and transverse directions using $6.35 \mathrm{~mm}$ high, $3.2 \mathrm{~mm}$ diameter cylindrical specimens and through thickness compression was carried out on $3.2 \mathrm{~mm}$ diameter, $3.2 \mathrm{~mm}$ high cylinders. Plane strain compression tests were also performed using the procedures adopted by Wonciewicz and Backofen ${ }^{5}$ and by Kelley and Hosford ${ }^{6,7}$. All compression tests were carried out using incremental loading, with Teflon lubrication which was renewed for each increment. Both tensile and compressive tests were performed on an Instron
$10,000 \mathrm{~kg}$ machine using a strain rate of $2.5 \times 10^{-4} /$ sec.

Preferred orientations were determined for samples taken from positions approximately $3 \mathrm{~m}$ and $6 \mathrm{~m}$ from the front end of the extrusion using the back reflection method on composite samples cut to reveal a plane whose normal makes an angle of $54.7^{\circ}$ with the extrusion, transverse and thickness directions. This sampling procedure, which is described by Elias and Heckler ${ }^{3}$, allows a full quadrant of the pole figure to be constructed from back-reflection data alone. (0002), (10ī0), (1011) and (10ĩ) pole figures were prepared and pole figure inversion was carried out, using the computer programme devised by Morris and Heckler ${ }^{9}$, to give the crystal orientation distribution, which is the only complete description of the preferred orientation.

\section{RESULTS}

The preferred orientations determined from the two positions in each of the extrusions showed only minor differences along the length of the extrusions but there were major differences between the hot and cold extrusions. (0002) and (1010) pole figures are shown in Figure 2 for the cold extrusion and in Figure 3 for the hot extrusion. Both materials have substantial components in which the basal poles are tilted approximately $15^{\circ}$ away from the through thickness direction towards the extrusion direction but the hot extruded material has, in addition to these, components with the basal poles normal to the extrusion direction and almost parallel to the transverse direction.

The crystal orientation distribution functions shown in Figure 4, for the cold extruded, and in Figure 5, for the hot extruded materials, are obtained from inversion of the (0002), (1010) and (10i1) pole figures; the (1012) pole figure allows the statistical reliability of the data to be established. For both Figures 4 and 5 the standard error is approximately \pm 1 random. The crystal orientation distributions are plotted in spherical co-ordinates, the intensity level at a given point $(\psi, \theta, \phi)$ in the 


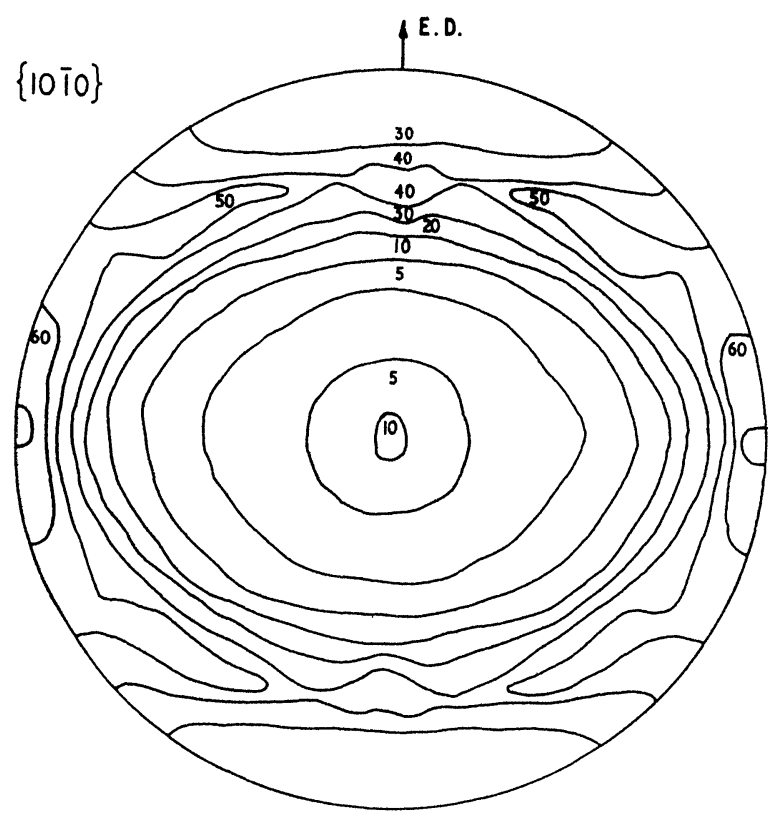

The data of Figures 4 and 5 show that the textures are comprised of almost pure fibre components with the component having the basal pole $\sim 15^{\circ}$ from the through thickness direction being more strongly developed in the cold extrusion than in the hot extrusion. The component having basal poles near to the transverse direction, which is present in the hot extruded but not the cold extruded material, is the least fibrous of the main features of this texture. There is clear indication of the
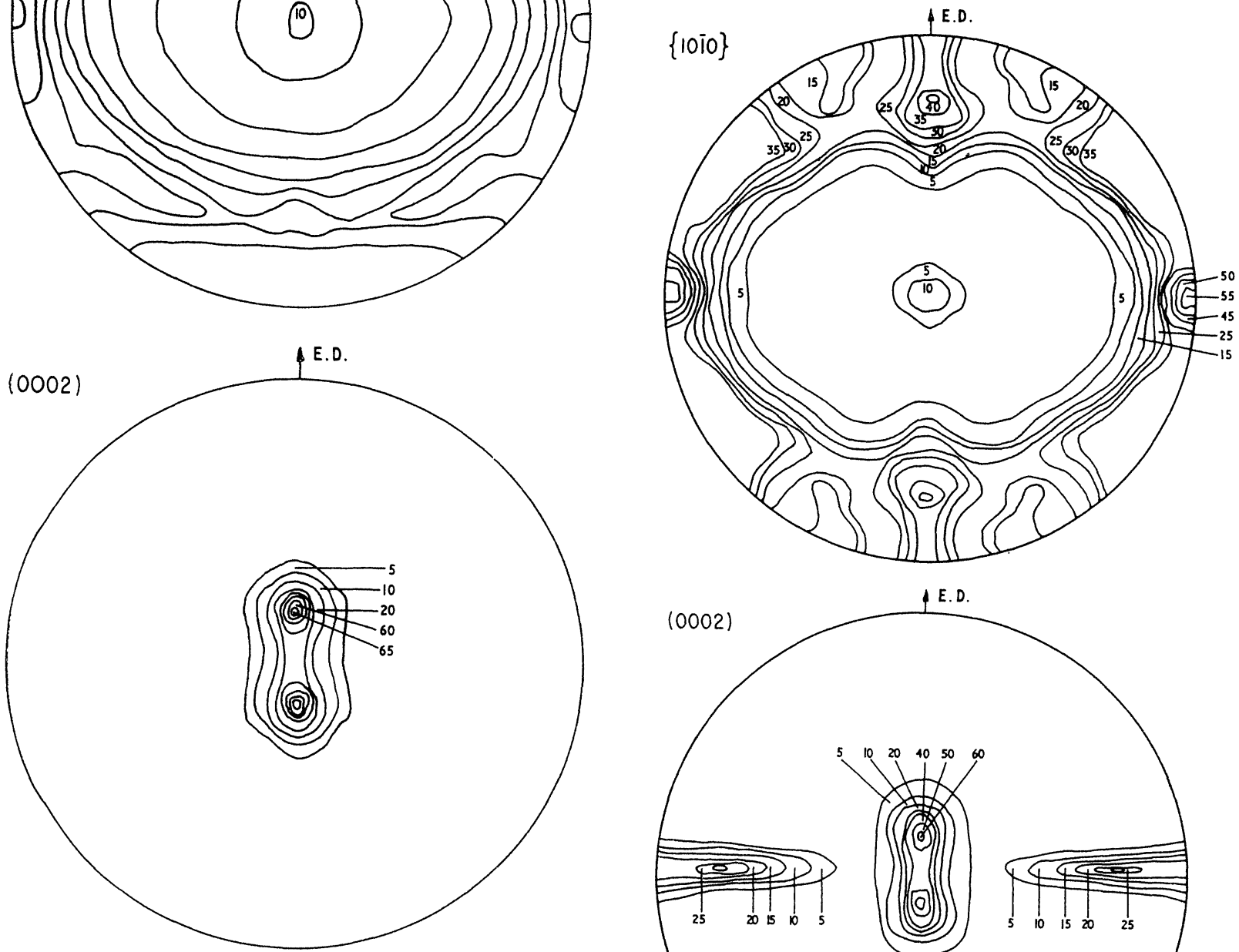

FIGURE $2\{10 \overline{10}\}$ and (002) pole figures for the cold extruded and annealed magnesium. The units are arbitrary, background levels are 0.5 and 4.0 for the (0002) and $\{1010\}$ pole figures respectively.

figures being the multiple of the physical volume of material in a random sample whose orientation is defined by the three angles $\psi, \theta, \phi$. The relationship between $(\psi, \theta, \phi)$ and the (hkil) [uvtw] MillerBravais index representation of crystal orientation is shown stereographically in Figure 6.

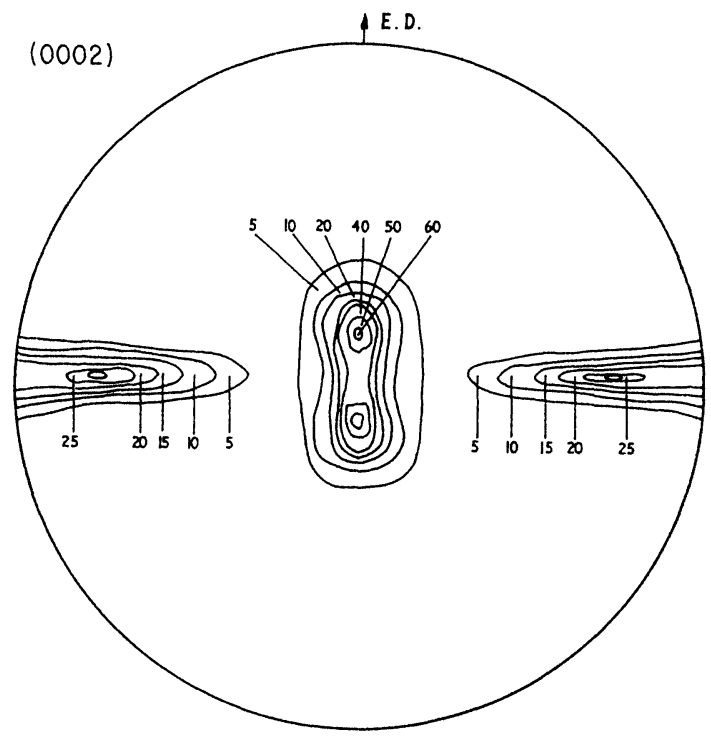

FIGURE $3 \quad\{10 \overline{1} 0\}$ and (0002) pole figures for the hot extruded and annealed magnesium. A background level of 0.5 applies to both figures. 
$\{10 \overline{1} 0\}\langle 11 \overline{2} 0\rangle$ type of component at $\psi, \theta, \phi$ co-ordinates $(90,90,0)$ and $(90,90,60)$ being more prevalent than the $\{11 \overline{2} 0\}\langle 10 \overline{1} 0\rangle$ at co-ordinates $(90,90,30)$. Both hot and cold extruded materials show an (0001) [uvt0] fibre texture component, fairly symmetrically spread, merging with the component which has the [0001] fibre axis $15^{\circ}$ from the through thickness direction and perpendicular to the transverse direction.

The mechanical property data are shown in Figures 7 and 8 for the cold and the hot extrusions respectively. These are in the form of initial yield loci in plane stress sections. A yield locus shows the levels of the applied stresses at which the material becomes plastic and its definition assumes a sharp transition, the yield point, from the elastic to the plastic state. In practice a yield point of this type does not occur and the loci of Figures 7 and 8 are plotted for a largest principal strain of 0.02 .

An important property of the yield locus is that the tangent plane to the locus at a given point defines the ratio of strain increments which result on yielding under the stress state represented by that point. If the line joining the origin to the yield locus at the particular point under consideration is termed the stress vector, the corresponding strain increment vector is defined in its components, referred to strain increment axes parallel to the corresponding stress axes, by the outward pointing normal to the yield locus at the point of termination of the stress vector. The yield locus is thus defined by two sets of information, the yield stress, defining

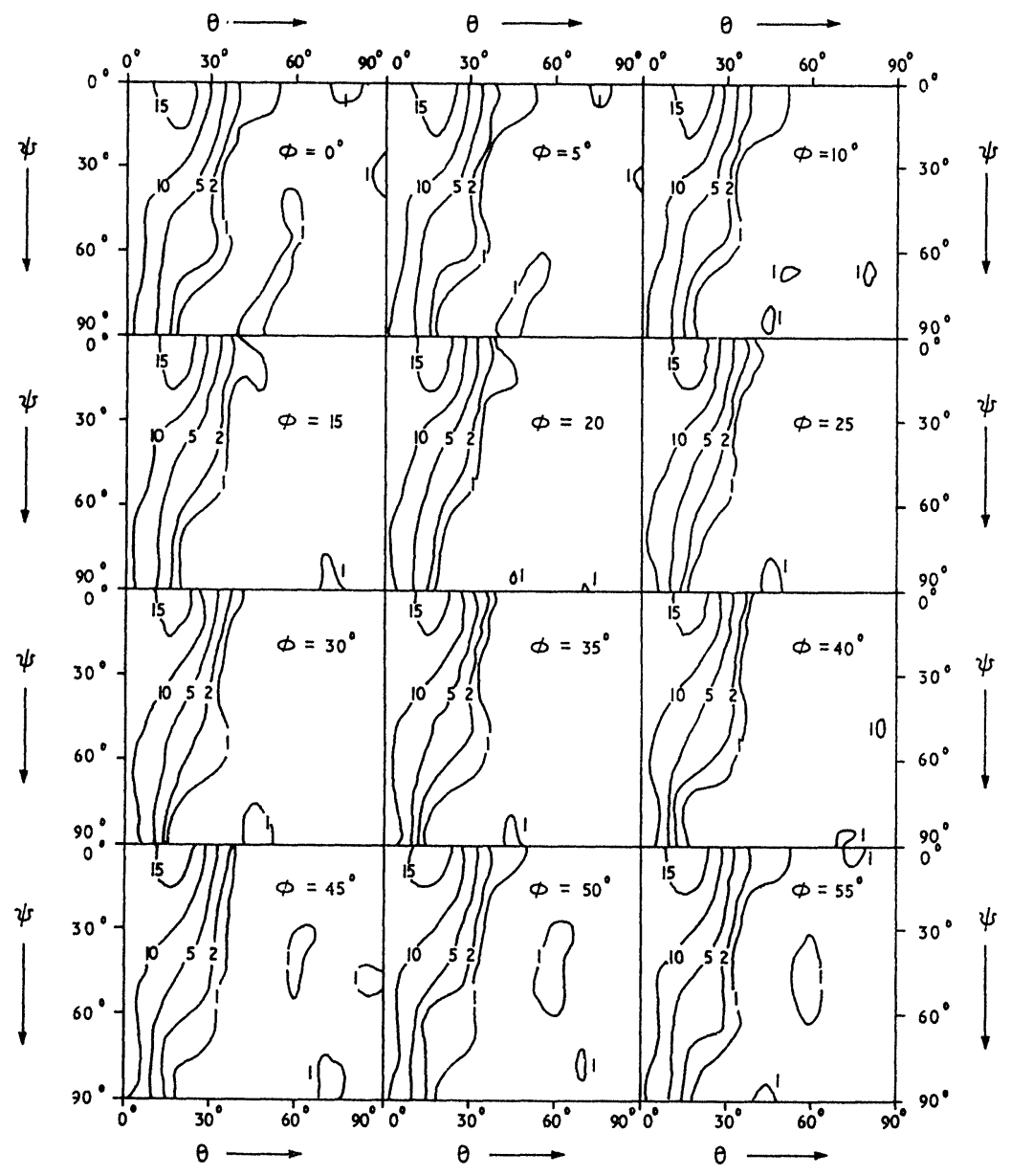

FIGURE 4 Crystallite orientation distribution function for the cold extrusion. The contours show the volume representation as a multiple of that for a random sample at a point in a three dimensional space characterised by the three spherical co-ordinates (Euler angles) $(\psi, \theta, \phi)$. The constant $\phi$ sections should be regarded as stacked in sequence to make a rectangular box of dimensions $90^{\circ} \times 90^{\circ} \times 60^{\circ}$. 
the distance of the locus from the origin and the strain ratios which give information about the local slope of the yield locus. Plane strain comcompression tests define the level of the largest principal stress corresponding to the points of horizontal and vertical tangency to the yield locus.

A major problem in defining the shape of the yield locus for hexagonal metals is that the strain ratio can vary rapidly with strain, or correspondingly for conditions of imposed constant strain ratio the applied stress may vary with strain. This is demonstrated in Figure 9 for uniaxial tension testing of the cold extruded material parallel to the extrusion direction; the strain ratio is seen to vary over a wide range in a small longitudinal strain increment. For this reason, linked with the absence of a sharp yield point, it is pointless to seek a more accurate means of plotting the yield locus than that used here of selecting a small arbitrary strain increment at which to choose the stress level and the strain ratio.

Two sets of data are recorded in both Figure 7 and Figure 8; the open symbols relate to results obtained from samples taken approximately $3 \mathrm{~m}$ from the front end of the extrusion and the filled symbols are for samples taken approximately $6 \mathrm{~m}$ from the front end. In each case the point is the average from duplicate tests. It is to be expected that the results from the two sections will be at variance in view of the grain size variation along the length of the extrusion shown in Figure 1. The grain size variation is strongest in the cold extruded material, and as a result the mechanical property variation is also greatest for this material.

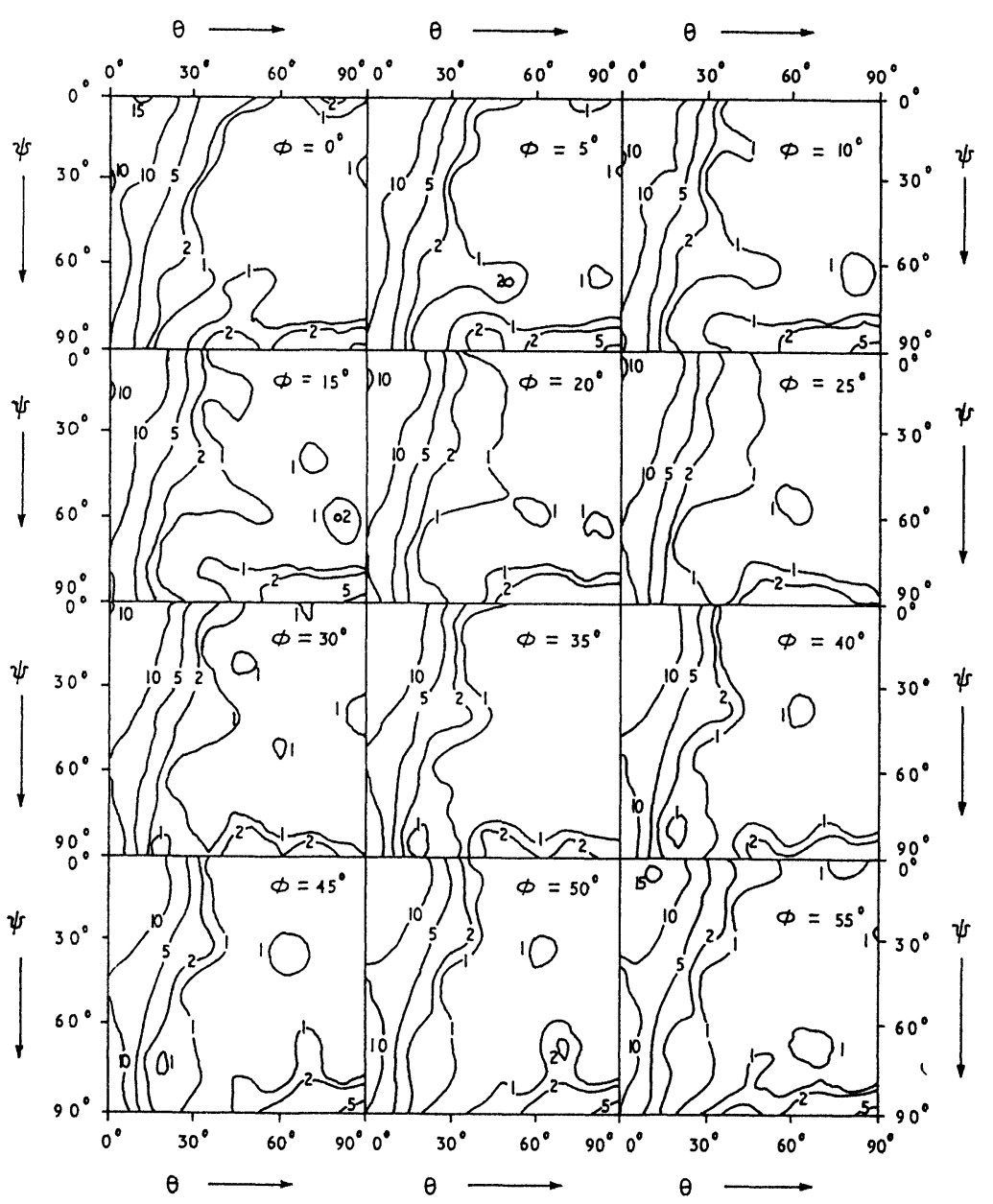

FIGURE 5 Crystallite orientation distribution function for the hot extrusion. The similarities with the cold extrusion texture are obvious, but an additional component at $\psi=90, \theta=90, \phi=0-60^{\circ}$ is seen in the hot extrusion. 


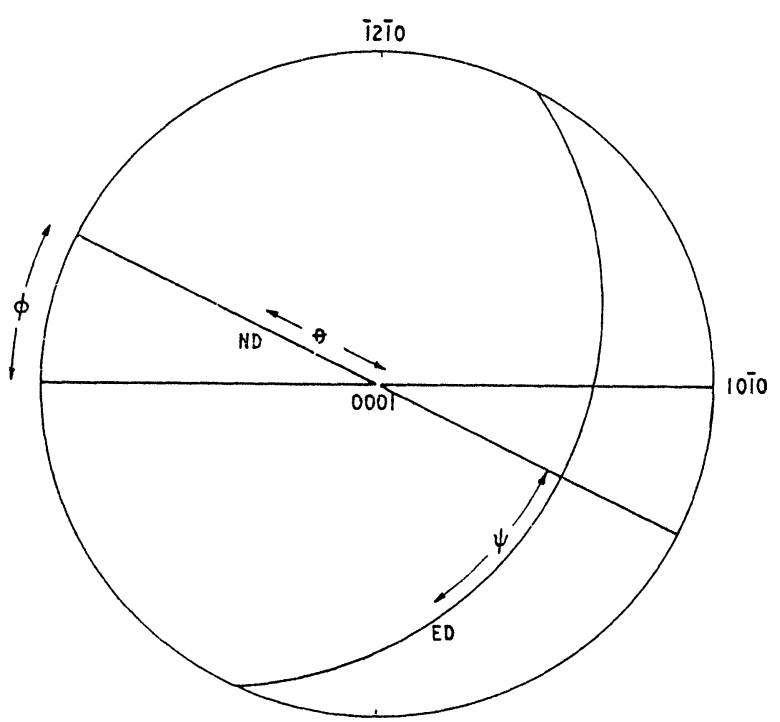

FIGIJRE 6 Definition of spherical angles $(\psi, \theta, \phi)$ relative to the orthogonal crystal axes in Miller.Bravais indices. ND is the normal to the plane of the extrusion, ED is the extrusion direction.

The range of grain sizes is not large enough to determine the grain size dependence of the yield stress, and it must be remarked that a soft material with a high work hardening rate like magnesium is susceptible to any slight mishandling and is likely to show appreciable scatter in the results, although every care was taken to avoid damaging the samples in preparation. The main object here is to compare the texture hardening under different stress states associated with the two textures under consideration. The full lines drawn in Figures 7 and 8 thus represent the average yield loci for the two materials, corresponding to a grain size for both materials, of about $45 \mu$. Any differences between the two yield loci are attributable to texture differences.

The salient features are that both materials are stronger in tension than in compression, where the stresses are applied in the plane of the extrusion. The hot extrusion is marginally stronger under tension applied parallel to the extrusion direction than when the tensile stress is applied along the transverse direction; the converse is true for the cold extrusion. Under compressive stress states the transverse direction is stronger for the hot extrusion and the two directions have approximately equal strength in the cold extrusion. The strength of the hot extrusion is greater than that of the cold extrusion for all states of stress.

\section{DISCUSSION}

There are two aspects of the present work which warrant discussion; the development of the textures in the two cases and the relationship between texture and mechanical properties. Both of these questions revolve around the available deformation mechanisms and some preliminary discussion of these is necessary.

At room temperature magnesium deforms by basal slip with a $\langle 11 \overline{2} 0\rangle$ vector, by $\{10 \overline{1} 2\}$ twinning and by $\{10 \overline{1} 1\}$ twinning. $\{10 \overline{1} 3\}$ twinning also occurs but with less frequency than $\{10 \overline{1} 1\}$ and there have been unsubstantiated reports of $\{11 \overline{2} x\}$ twins. The $\{11 \overline{2} x\}$ twins are common in titanium and zirconium but Wonciewicz and Backofen ${ }^{5}$ found no evidence of them in magnesium under conditions most favourable to their development.

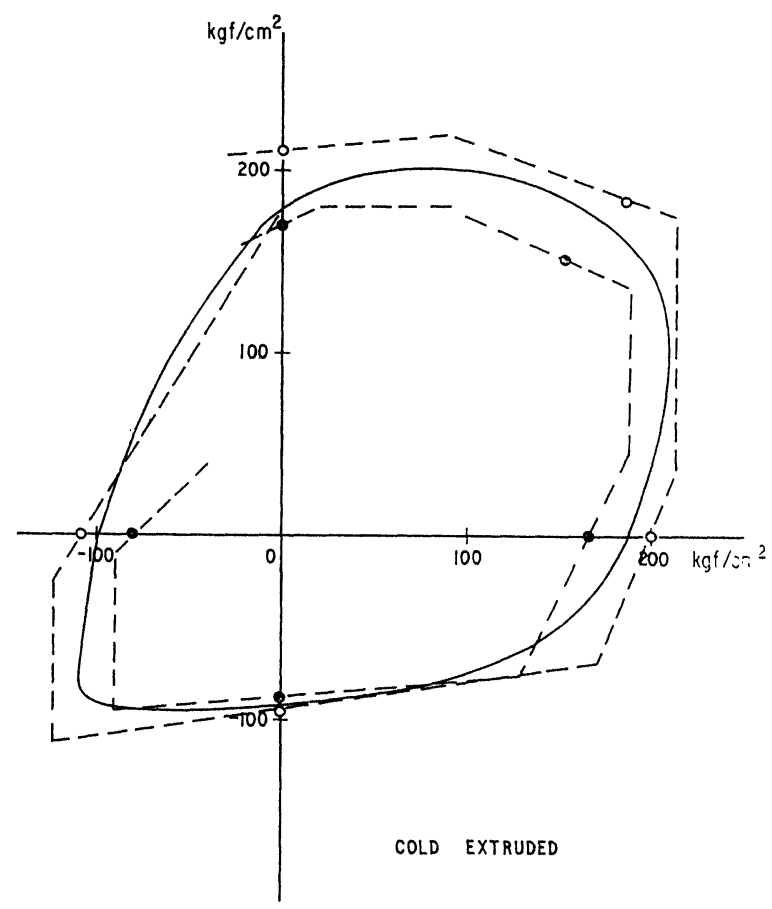

FIGURE 7 Experimental yield locus for the cold extruded material, obtained by the procedures used by Kelley and Hosford ${ }^{7}$. The filled points show stress levels at 0.02 strain for material taken $6 \mathrm{~m}$ from the front of the extrusion, the open points are from the $3-\mathrm{m}$ position. The dotted lines are derived from strain ratio measurements and are indicative of the slope of the yield locus through the appropriate point. Dotted lines which are perpendicular to the stress axes and which do not pass through a datum point show the results of plane strain compression tests. 


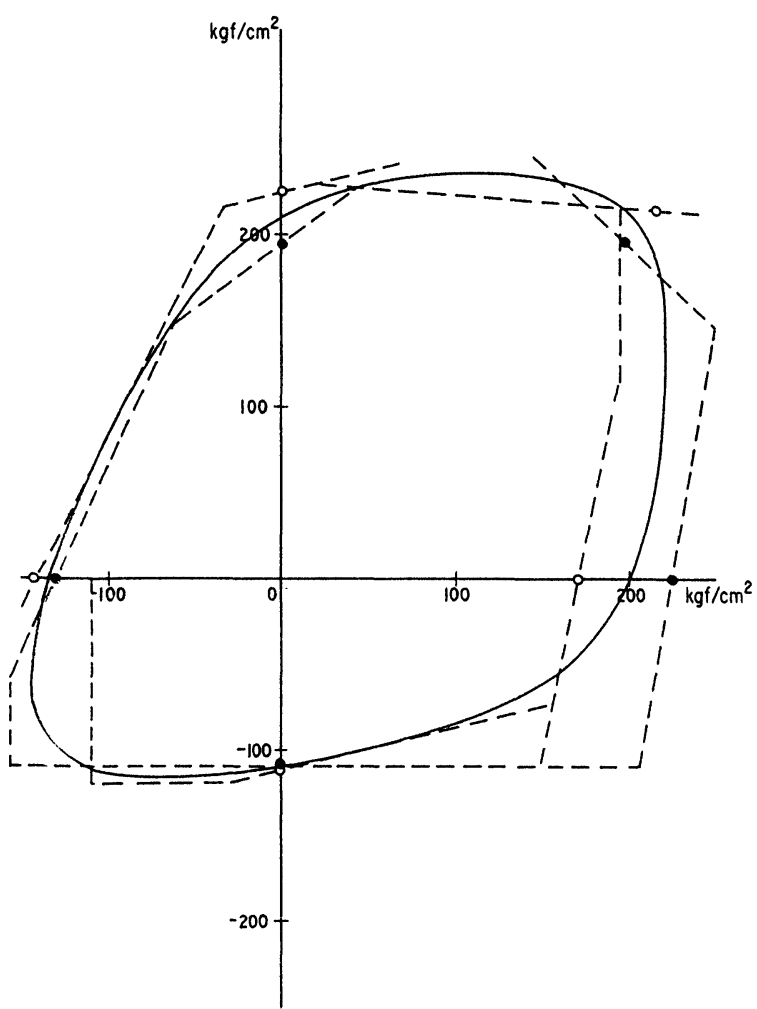

FIGURE 8 Experimental yield locus for the hot extruded material.

$\{10 \overline{1} 0\}\langle 1 \overline{2} 10\rangle$ and $\{10 \overline{1} 1\}\langle 1 \overline{2} 10\rangle$ slip are reported to occur ${ }^{10}$ in magnesium deformed at elevated temperatures, and this has been proposed as accounting for the ductility transition in magnesium which occurs at about $200^{\circ} \mathrm{C}$.

In the deformation of polycrystals the maintenance of continuity of material across grain boundaries requires that there are at least five independent deformation modes available to satisfy the five independent components of the strain tensor. ${ }^{11}$ In Table II the contribution to the strain components by the various deformation modes reported to occur in magnesium are listed. $\{10 \overline{1} 3\}$ twinning is ignored, since it is reported to occur only to a small extent and because it contributes no strain components not already covered by $\{10 \overline{1} 1\}$ twinning.

Slip with a $\langle 11 \overline{2} 0\rangle$ vector makes no contribution to $d \varepsilon_{33}$ (the normal strain along the c-axis) $\{10 \overline{1} 2\}$ twinning causes only expansion along the c-axis and $\{10 \overline{1} 1\}$ twinning results in contraction along the c-axis. $\{10 \overline{1} 1\}$ slip makes no contribution to the strains additional to that available from
$\{10 \overline{1} 0\}$ and $\{0001\}$ slip. The five independent deformation modes may thus be chosen from $\{10 \overline{1} 0\}$ and $\{0001\}$ slip and $\{10 \overline{1} 2\}$ and $\{10 \overline{1} 1\}$ twinning. It must always be remembered, however, that twinning causes major reorientation of the twinned part of the crystal and the total strain caused by twinning an entire crystal is in invariant quantity. That the contribution of modes listed above can satisfy five independent strain components should not, therefore, be taken to indicate that the material is capable of homogeneous deformation: the deformation will most probably be highly inhomogeneous, with a given crystal approximating to the imposed strain by separate portions of the crystal deforming in different ways to achieve, on average, the required shape change.

It is unlikely, because of the rapid crystal fragmentation and discontinuous reorientation, that a complete theory of the plasticity of hexagonal metals will be formulated. Texture development in these metals can thus be accounted for only qualitatively and it is not rewarding to try to account for the strength at strains beyond the initial yield.

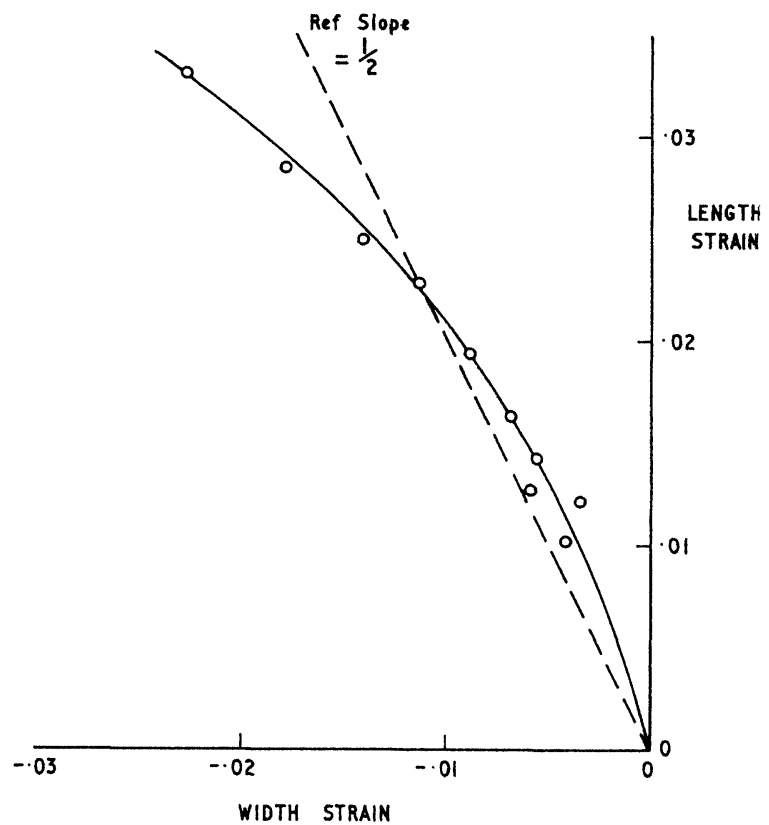

FIGURE 9 Plastic length strain versus plastic width strain plotted for the cold extruded material tested in tension parallel to the extrusion direction. This shows clearly the wide variation in strain ratio after yielding. The reference slope of $\frac{1}{2}$ would be followed by an isotropic material. 
TABLE II

\begin{tabular}{|c|c|c|c|c|c|c|c|}
\hline & \multirow{2}{*}{$\begin{array}{l}\text { Shear } \\
\text { mode }\end{array}$} & \multicolumn{6}{|c|}{ Strain components } \\
\hline & & $d \varepsilon_{11}$ & $d \varepsilon_{22}$ & $d \varepsilon_{33}$ & $d \varepsilon_{12}$ & $d \varepsilon_{23}$ & $d \varepsilon_{31}$ \\
\hline 1 & $(0001)[1150]$ & 0.0000 & 0.0000 & 0.0000 & 0.0000 & 0.0000 & 0.5000 \\
\hline 2 & $(0001)[1210]$ & 0.0000 & 0.0000 & 0.0000 & 0.0000 & -0.4330 & 0.2500 \\
\hline 3 & $(0001)[2110]$ & 0.0000 & 0.0000 & 0.0000 & 0.0000 & 0.4330 & 0.2500 \\
\hline 4 & $(1100)[11 \overline{2} 0]$ & 0.0000 & 0.0000 & 0.0000 & 0.5000 & 0.0000 & 0.0000 \\
\hline 5 & $(10 \overline{1} 0)[1210]$ & -0.4330 & 0.4330 & 0.0000 & 0.2500 & 0.0000 & 0.0000 \\
\hline 6 & $(0110)[2110]$ & 0.4330 & -0.4330 & 0.0000 & 0.2500 & 0.0000 & 0.0000 \\
\hline 7 & $(1 \overline{101})[1120]$ & 0.0000 & 0.0000 & 0.0000 & -0.4409 & 0.0000 & -0.2357 \\
\hline 8 & (1011) [1210] & -0.3818 & 0.3818 & 0.0000 & 0.2205 & 0.2041 & -0.1178 \\
\hline 9 & (0111) [2110] & 0.3818 & -0.3818 & 0.0000 & 0.2205 & 0.2041 & 0.1178 \\
\hline 10 & (0111) [2110] & 0.3818 & -0.3818 & 0.0000 & 0.2205 & -0.2041 & -0.1178 \\
\hline 11 & (1011) [1210] & -0.3818 & 0.3818 & 0.0000 & 0.2205 & -0.2041 & 0.1178 \\
\hline 12 & (1101) [1120] & 0.0000 & 0.0000 & 0.0000 & -0.4409 & 0.0000 & 0.2357 \\
\hline 13 & $(1 \overline{1} 01)$ [1102] & 0.0000 & +0.4157 & -0.4157 & 0.0000 & -0.2777 & 0.0000 \\
\hline 14 & (1011) [1012] & +0.3118 & +0.1038 & -0.4157 & +0.1800 & -0.1388 & -0.2405 \\
\hline 15 & (0111) [0I12] & +0.3118 & +0.1038 & -0.4157 & -0.1800 & +0.1388 & -0.2405 \\
\hline 16 & (0I11) [01I2] & +0.3118 & +0.1038 & -0.4157 & -0.1800 & -0.1388 & +0.2405 \\
\hline 17 & (1011) [1012] & +0.3118 & +0.1038 & -0.4157 & +0.1800 & +0.1388 & +0.2405 \\
\hline 18 & (1101) [1102] & 0.0000 & +0.4157 & -0.4157 & 0.0000 & +0.2777 & 0.0000 \\
\hline 19 & (1012) [1011] & -0.3741 & -0.1247 & +0.4988 & -0.2160 & 0.0166 & 0.0288 \\
\hline 20 & (1102) [1101] & 0.0000 & -0.4988 & +0.4988 & 0.0000 & 0.0668 & 0.0000 \\
\hline 21 & $(0112)[0111]$ & -0.3741 & -0.1247 & +0.4988 & 0.2160 & -0.0166 & 0.0288 \\
\hline 22 & (0112) [0111] & -0.3741 & -0.1247 & +0.4988 & 0.2160 & 0.0166 & -0.0288 \\
\hline 23 & $(1012)[1011]$ & -0.3741 & -0.1247 & +0.4988 & -0.2160 & -0.0166 & -0.0288 \\
\hline 24 & (1102) [1101] & 0.0000 & -0.4988 & +0.4988 & 0.0000 & -0.0668 & 0.0000 \\
\hline
\end{tabular}

Strain components referred to axes parallel to $1-1120,2-1 \overline{1} 00$ and 3-0001 due to slip and twinning on the systems indicated. The strains are calculated for $c / a=1.62$.

The textures developed in cold extrusion can be accounted for on the assumption that basal slip, $\{10 \overline{1} 2\}$ and $\{10 \overline{1} 1\}$ twinning are the operative deformation modes. It is assumed here that the strain state in extrusion is plane strain; this is an approximation. It should also be recognised that the textures presented in Figures 2, 3, 4, and 5 are for the annealed material. However, all available evidence indicates that the basal pole distribution is essentially unaltered by recrystallisation, ${ }^{12}$ the most commonly quoted reorientation on recrystallising is a $30^{\circ}$ rotation about the basal pole and since the results of Figure 4 indicate that for the cold extruded material the texture is composed of basal pole fibre textures it is probable that the deformation texture was similar to the annealing texture in all respects. It is thus necessary to consider only the basal pole reorientation during deformation.

$\{0001\}$ slip tends to reorient the basal plane towards a position of parallelism with the extrusion plane; $\{10 \overline{1} 2\}$ twinning causes an $86.3^{\circ}$ reorientation and $\{10 \overline{1} 1\}$ twinning a $56^{\circ}$ reorientation about a $\langle 11 \overline{2} 0\rangle$ as axis. To meet the requirements of plane strain deformation the twin system which will be most favoured is the one with the $\langle 11 \overline{2} 0\rangle$ lying closest to the transverse direction. Basal slip thus tends to focus the basal poles towards the extrusion plane pole and twinning favours reorientation about the transverse direction as axis. $\{10 \overline{1} 2\}$ twinning occurs for basal poles lying outside a great circle containing the transverse direction and at $45^{\circ}$ to the extrusion plane, i.e. for orientations requiring a positive c-axis strain, $\{10 \overline{1} 1\}$ twinning occurs for basal poles lying inside this great circle for orientations requiring a negative $\mathrm{c}$-axis strain.

The approximately $15^{\circ}$ misorientation of the basal poles from the extrusion plane normal is a natural consequence of the difference in the reorientation between the two twin modes together with the tendency of basal slip to rotate basal poles towards the extrusion plane normal. $\{10 \overline{1} 2\}$ twinning occurs much more readily than the $\{10 \overline{1} 1\}$ twinning so that material within a $\{10 \overline{1} 1\}$ twin is soft compared with the surrounding matrix. 
$\{10 \overline{1} 1\}$ twinning will thus be followed immediately by $\{10 \overline{1} 2\}$ twinning and the basal poles concentrate near the centre of the pole figure (Figure 2).

The central basal pole distribution in the hot extruded material is accounted for in exactly the same way as for the cold extruded texture. The basal poles close to the transverse direction are not accounted for in this way, however. This distribution can be accounted for by $\{10 \overline{1} 0\}$ slip or by $\{10 \overline{1} 1\}$ slip either, or both, of which may be expected to be available for the case of hot deformation but not for cold deformation. The following discussion will assume that $\{10 \overline{1} 0\}$ slip is the operative mode but the main features would be the same, for $\{10 \overline{1} 1\}[1 \overline{2} 10]$ and $(10 \overline{1} \overline{1})[1 \overline{2} 10]$ is vectorially equivalent to single $(10 \overline{1} 0)$ [12 10$]$ slip.

$\{10 \overline{1} 0\}$ slip is the preferred deformation mode for crystals with their basal pole parallel to the transverse direction. For such crystals there is no tendency for basal pole rotation since the basal pole is the rotation axis. The rotation for these orientations can be very easily computed, after the procedure of Dillamore et $a l_{.}{ }^{13}$; they are in fact identical in character to the rotations for cubic crystals with a $\langle 111\rangle$ transverse direction and are shown in Figure 10. $d \phi / d E$ is the angular rotation in radians per unit natural plane strain, $\phi$ is the

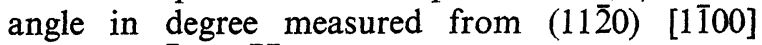
towards (1100) [11120]. Zero $d \phi / d E$ indicates a null rotation orientation, positive $d \phi / d E$ indicates a rotation which increases $\phi$, negative $d \phi / d E$ decreases $\phi$. A positive slope at zero $d \phi / d E$ to the plot of Figure 10 corresponds with a metastable orientation, a negative slope to a stable orientation. Thus $(11 \overline{2} 0)$ [1100] is a stable orientation; ( $1 \overline{1} 00$ [11120] is metastable and is expected to split on deformation and rotate to the symmetrical stable orientations $(1 \overline{2} 10)$ [ $\overline{1} 010]$ and $(2 \overline{1} 10)$ [0 $\overline{1} 10]$, which are both $30^{\circ}$ away. It will be recalled that the results presented in Figure 5 indicate that the $\{1 \overline{1} 00\}\langle 11 \overline{2} 0\rangle$ type of orientation is more prevalent than the $\{11 \overline{2} 0\}$ $\langle 1 \overline{1} 00\rangle$ type in the hot extruded recrystallisation texture. This is consistent with the $30^{\circ}$ rotation about $\langle 0001\rangle$, the relationship between deformation and recrystallisation textures reported for hexagonal metals.

In the present case there are also grounds for the existence of a nucleation mechanism consistent with the $30^{\circ}$ rotation about $\langle 0001\rangle$. A metastable orientation such as (1100) [11120] will, if orientation splitting occurs, be ideally situated at the centre of a region of pronounced lattice curvature to provide

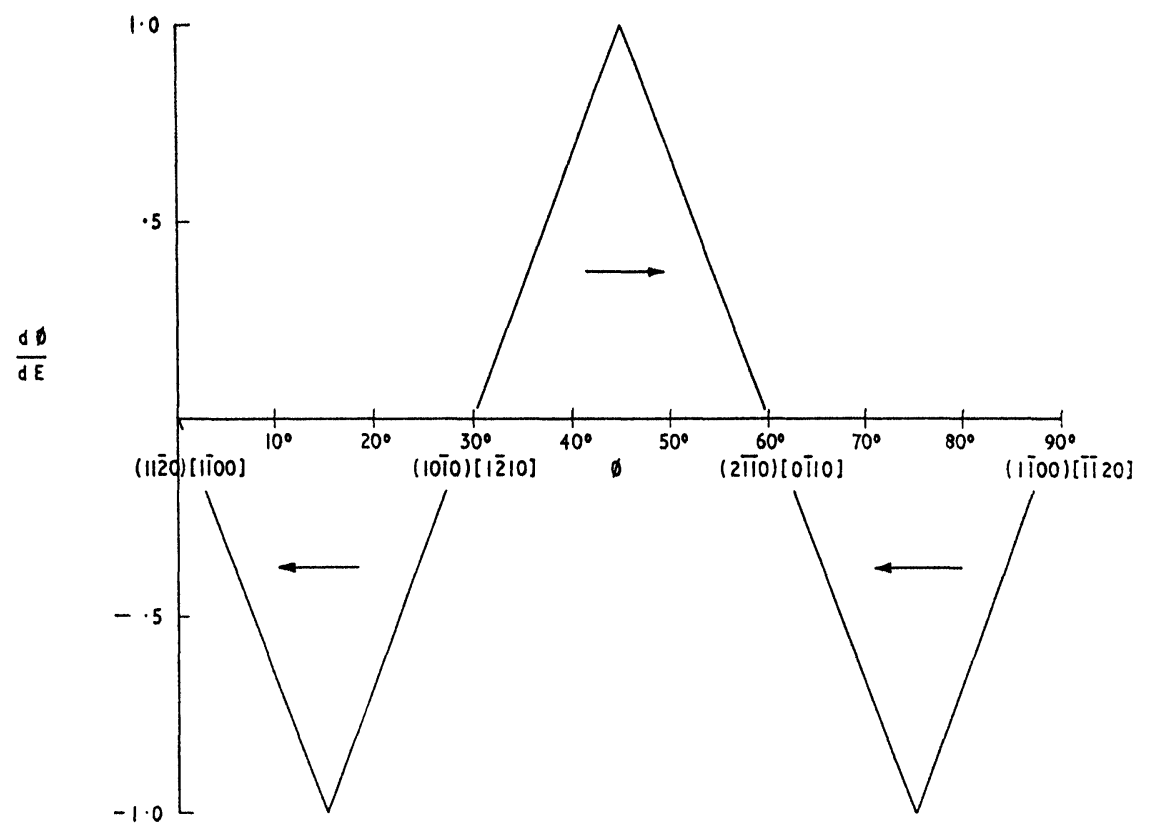

FIGURE 10 The instantaneous rate of crystal rotation $d \phi / d E$ plotted against angular location $\phi$ from (1120) [1100] of the orientations with [0001] transverse direction deformed in plane strain and deforming by $\{10 \overline{1} 0\}$ slip. Orientations of the type $\{11 \overline{2} 0\}\langle\overline{1} 100\rangle$ are stable, those of the $\{1100\}\langle 1120\rangle$ are metastable and will tend to form transition bands by splitting to the symmetrically disposed stable components. 
recrystallisation nuclei. Recrystallisation from such regions has been demonstrated by $\mathrm{Hu}^{14}$ and Walter and Koch $^{15}$.

There is obviously no c-axis strain for the family of orientations just considered and $\{10 \overline{1} 0\}$ slip can provide the imposed strain in its entirety. Consider now the c-axis to be progressively moved away from the transverse direction; Figure 11 shows the c-axis strain as a function of the position of the basal pole relative to the specimen axes. The dotted line is shown as a crude indicator of the basal pole locations for which basal slip and prism slip would most effectively contribute to the nonbasal strain components; to the left of the dotted line basal slip would, on average, be preferred; to the right, prism slip would be more likely to be effective. For positive c-axis strains $\{10 \overline{1} 2\}$ twinning operates, for negative c-axis strain $\{10 \overline{1} 1\}$ twinning occurs.

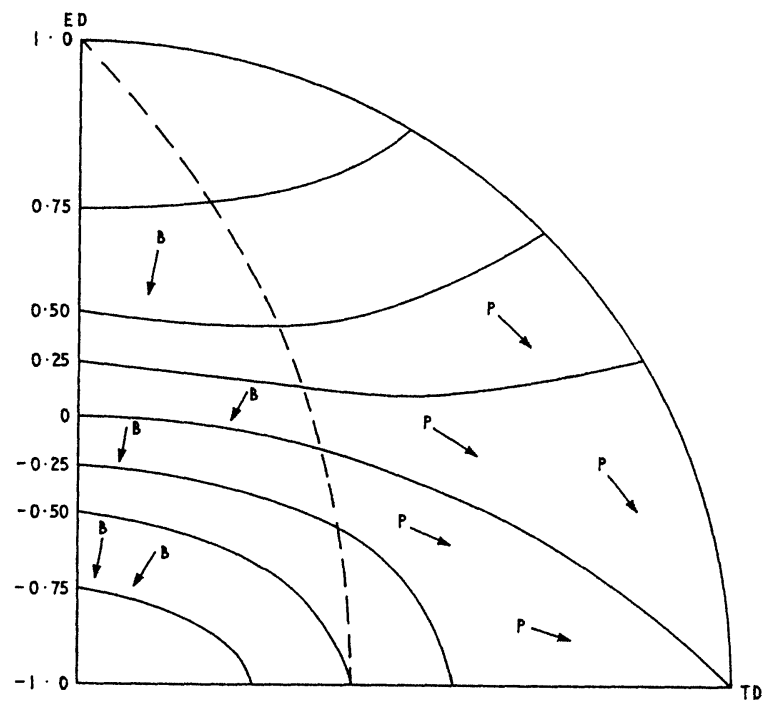

FIGURE 11 Stereographic plot of the normal strain perpendicular to the basal plane as a function of the location of the basal pole relative to the physical axes. The dotted line is a crude indicator of the positions of basal poles for which basal slip and $\{10 \overline{1} 0\}$ slip are approximately equally capable of contributing to the strain. Arrows labelled B show the sense of basal pole rotations when basal slip predominates, those labelled $\mathrm{P}$ relate to rotations when $\{1010\}$ slip is active.

The arrows marked $\mathrm{B}$ indicate the general trend of basal slip rotations, those marked $\mathrm{P}$ show the sense of basal pole rotations when prism slip occurs.

As mentioned previously twinning tends to re-orient material so that the basal poles lie near the centre of the pole figure but it can be seen that where prism slip is preferred there is less need for twinning to occur than where basal slip is preferred, by virtue of the lower overall level of c-axis strain on the right hand side of the dotted line of Figure 11. There will still be loss of material from this region as a result of the twinning but when $\{10 \overline{1} 0\}$ slip operates, causing the untwinned volume to rotate so as to align the $\{0001\}$ poles with the transverse direction, the depletion will be incomplete. Since $\{10 \overline{1} 1\}$ twinning operates only at high stress levels it may not be favoured to contribute when the required c-axis strain is small and positive; if the surrounding material accommodates these grains the spread of basal poles along the equator of the pole figure is thus accounted for.

At low temperature Kelley and Hosford ${ }^{6}$ have shown that the strain components most readily matched by slip on $\{10 \overline{1} 0\}$ have to be accommodated by combined $\{10 \overline{1} 2\}$ and $\{10 \overline{1} 1\}$ twinning, there will in this case be gross reorientation removing the basal poles initially parallel to the transverse direction away from this position.

The differences between the hot and cold extrusion textures are thus explained by the operation of $\{10 \overline{1} 0\}$ slip at the high temperature. It now remains to consider what effects the differences in texture have on the mechanical behaviour.

The principle objective of polycrystalline plasticity theory is to account for the properties of polycrystalline aggregates in terms of the properties of the constituent single crystals. In cubic metals deforming by slip on only one set of crystallographically equivalent systems, it is reasonable for the theory to attempt to relate the properties of the polycrystal to the contributions to deformation of the different slip systems. This approach is not viable for hexagonal metals which deform on twin systems as well as on slip systems; the alternative approach of actually discovering the stress strain behaviour of individual crystals of all orientations and averaging over the range of orientations represented in the polycrystalline material under study is also unreasonable. It is possible to calculate for a given imposed strain the sum of the shears for each of the deformation modes averaged over the crystals represented in the sample under consideration. The stresses associated with the given state of strain can then be calculated if the critical resolved shear stresses for the various deformation modes are known. It is usually necessary to know the critical resolved shear stresses before the relative shear magnitudes can be assigned, however, this 
problem is circumvented in the case which applies here, where different deformation modes satisfy different components of the strain tensor.

In the present work the yield locus envelope defined by the plane strain tangents has been sought by computing the shear magnitudes, averaged over the crystallite orientation distribution using the data shown in Figures 4 and 5; the systems assumed to operate were $\{10 \overline{1} 2\}$ and $\{10 \overline{1} 1\}$ twinning and $\{10 \overline{1} 0\}$ and $\{0001\}$ slip. The computer programme first calculated the twin shear to match the imposed c-axis strain and selected the combination of twin systems which minimised the amount of slip required on $(10 \overline{1} 0\}$ systems. No attempt was made to minimise the basal shear, it being assumed that relative to the other modes the basal shear was essentially free.

Although in computation it was assumed that $\{10 \overline{1} 2\}$ and $\{10 \overline{1} 1\}$ twinning and $\{10 \overline{1} 0\}$ slip occurred, the net effect of the calculations is to find the c-axis extension, the c-axis contraction and the plane strain component of deformation perpendicular to the c-axis averaged over all of the crystals. Rather than assign critical resolved shear stress values to the individual deformation modes, the initial yield stresses for plane strain compression with c-axis contraction, c-axis extension and zero $c$-axis strain taken from the work of Kelley and Hosford were used in computing the theoretical plane strain yield envelopes for the two materials considered here. The results are shown in Figure 12; the curves of Figures 7 and 8 are replotted in Figure 12a for comparison with the theoretical yield envelopes of Figure 12b. The circled numbers alongside each branch of the theoretical yield envelope indicates the strain state corresponding to Table III, where the results used to produce Figure $12 \mathrm{~b}$ are tabulated in terms of calculated average shear strains on the three modes $\{10 \overline{1} 2\}$ and $\{10 \overline{1} 1\}$ twin, $\{10 \overline{1} 0\}$ slip. It must be emphasised, however, that it is not fundamental to Figure $12 \mathrm{~b}$ that these systems be assumed to operate. In the work of Kelley and Hosford ${ }^{6}$ and Wonciewicz and Backofen ${ }^{5}$ no evidence for $\{10 \overline{1} 0\}$ slip was found during room temperature deformation, but plane strain perpendicular to $\{0001\}$ was accommodated by combined $\{10 \overline{1} 2\}$ and $\{10 \overline{1} 1\}$ twinning.

The theoretical yield envelopes have all been normalised to make the largest stress level unity, so it is the general shape features of Figure 12b which should be compared with 12a. The theoretical assessment accurately reflects the greater tensile yield stress in the extrusion direction than in the transverse direction for the hot extruded, it also shows the converse for the cold extruded. In compression the theoretical envelope shows the transverse strength to be greater than that in the extrusion direction for the hot extruded material but rather smaller differences between the two directions for the cold extruded. All of these are in agreement with the experimental results. The theoretical envelope shows, in addition, the correct general shape; the ratio of lengths measured at $45^{\circ}$ to the principal stress axes are in the correct order for the two materials.

The agreement obtained is perhaps better than might be anticipated. It is generally agreed that there is no critical resolved shear stress for twinning ${ }^{5,16}$ so the approach used here is perhaps the most reasonable in that it avoids explicitly assuming a critical resolved shear stress. However, there is an assumption implicit in the present work which is analogous to the assumption of a critical resolved shear stress. This assumption is that the deformation can be divided into three components, $\mathrm{c}$-axis compression, plane strain perpendicular to the

TABLE III

Shear averages and the different deformation modes.

\begin{tabular}{|c|c|c|c|c|c|c|}
\hline \multirow{2}{*}{$\frac{\text { Material }}{\text { System }}$} & \multicolumn{3}{|c|}{ Cold extruded } & \multicolumn{3}{|c|}{ Hot extruded } \\
\hline & $\begin{array}{c}\{10 \overline{1} 2\} \\
\text { twin }\end{array}$ & $\begin{array}{c}\{10 I 1\} \\
\text { twin }\end{array}$ & $\begin{array}{c}\{10 \overline{1} 0\} \\
\text { slip }\end{array}$ & $\begin{array}{c}\{10 \mathrm{I} 2\} \\
\text { twin }\end{array}$ & $\begin{array}{c}\{10 \overline{1} 1\} \\
\text { twin }\end{array}$ & $\begin{array}{c}\{10 T 0\} \\
\text { slip }\end{array}$ \\
\hline \multicolumn{7}{|l|}{ Strain state } \\
\hline 1 & 0.1042 & 1.5000 & 0.5013 & 0.0926 & 1.1649 & 0.9164 \\
\hline 2 & 1.2600 & 0.1240 & 0.4951 & 0.9785 & 0.1102 & 0.9115 \\
\hline 3 & 0.1149 & 1.6555 & 0.6496 & 0.4798 & 1.1603 & 0.5541 \\
\hline 4 & 1.3906 & 0.1368 & 0.6417 & 0.9747 & 0.5711 & 0.5507 \\
\hline 5 & 0.2405 & 0.1436 & 1.7433 & 0.1734 & 0.6720 & 1.4281 \\
\hline 6 & 0.2863 & 1.7445 & 1.7445 & 0.5644 & 0.2065 & 1.4262 \\
\hline
\end{tabular}




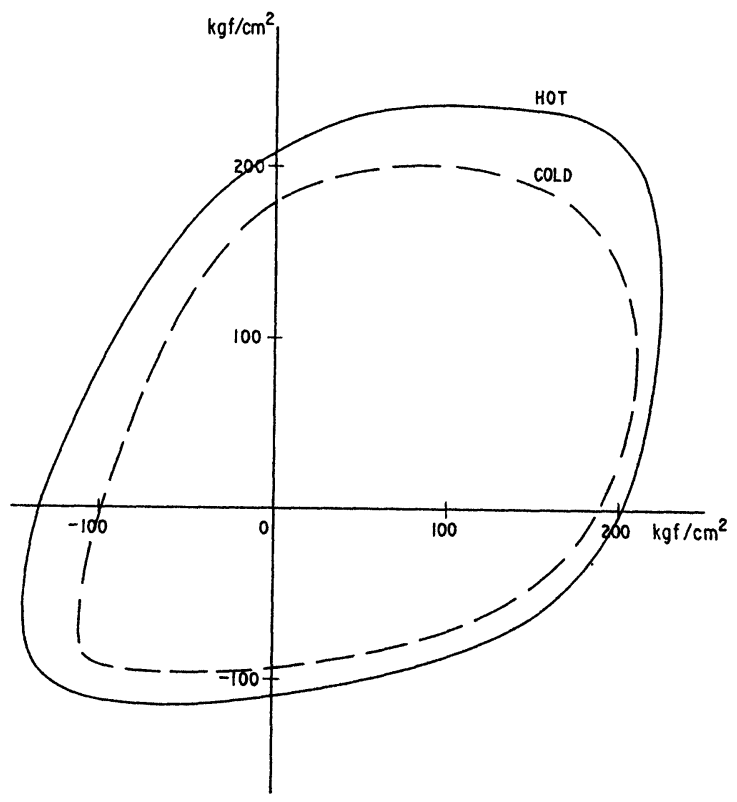

FIGURE 12a Yield loci for the hot and cold extruded materials, replotted without data points from Figures 7 and 8.

c-axis and shear in the basal plane, and that there is no interaction between these three components.

Wonciewicz and Backofen examined the question of the existence of a critical resolved shear stress for $\{10 \overline{1} 1\}$ twinning and found extreme values for the strain states corresponding to c-axis compression and plane strain perpendicular to the c-axis respectively. The good agreement between theory and experiment in the present work may depend on this result; the device of assuming $\{10 \overline{1} 0\}$ slip to operate has effectively allowed two critical resolved shear stresses for $\{10 \overline{1} 1\}$ twinning to be used. In the case of c-axis compression the shear stress for $\{10 \overline{1} 1\}$ twinning is that required when only the $\{10 \overline{1} 1\}$ mode operates, for plane strain perpendicular to the c-axis (equivalent to $\{10 \overline{1} 0\}$ slip) the shear stress for $\{10 \overline{1} 1\}$ twinning is that appropriate when co-operative $\{10 \overline{1} 2\}$ twinning occurs.

It is not appropriate to discuss here the question of the existence of a critical resolved shear stress, but the related question of the construction of flow loci which indicate the stresses to maintain flow at particular strain levels after yielding requires some comment. Kelley and Hosford have plotted flow loci at various strain levels after yield and have discussed them in a manner which suggests they have some physical significance. The only value of such loci is as a shorthand method of presenting incompletely the results of several different combined stress-strain experiments in one diagram. To link the points for each test to form a locus is misleading; the different points are physically unrelated. An initial yield locus has meaning in that it indicates the stress levels under which yielding would occur along different loading paths for a material in one initial condition. If two samples of this material are loaded along different loading paths to beyond the yield point, they develop different patterns of internal stress and are no longer in the same condition. For materials which twin extensively, not only is the stress pattern different for different loading paths, the crystal orientation distribution is also different. It becomes complicated beyond all possibility of detailed analysis to relate the stress-strain behaviour beyond initial yield for different loading paths in such materials, it also becomes improbable even if a critical resolved shear stress for twinning exists that it could be detected.

The very rapid change in material condition after yield is illustrated by the results shown in Figure 9. This shows the change in strain path which occurs for a constant loading path in magnesium (the cold extruded material tensile tested parallel to the

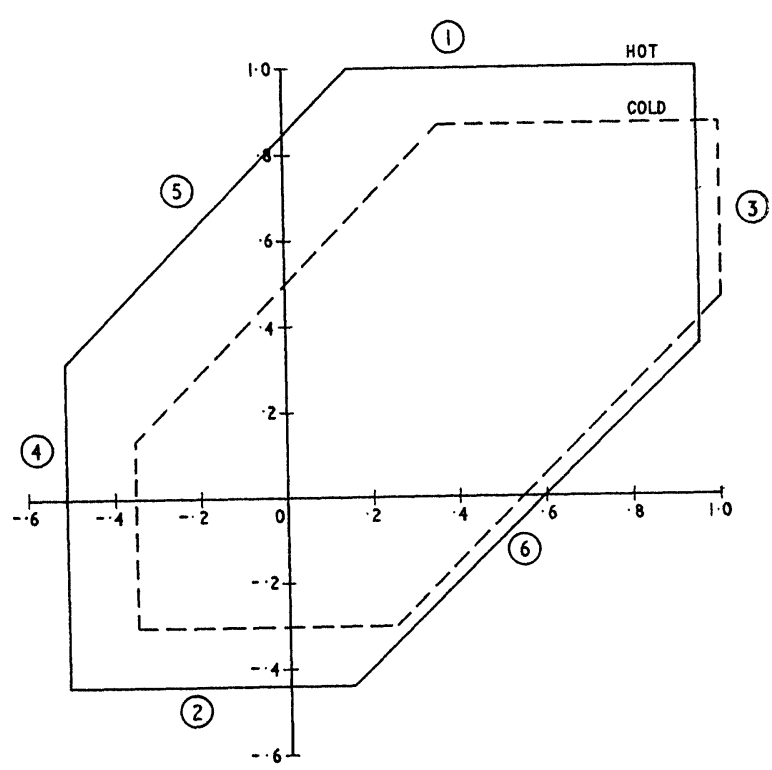

FIGURE 12b Theoretical yield loci computed from the data of Figures 4 and 5 on the assumption that c-axis strain, plane strain perpendicular to the c-axis and shear on the basal plane can be treated separately. The strengths for these three ideal deformations were taken from the work of Kelley and Hosford ${ }^{6}$. 
extrusion direction). A similar rapid change in stress path would be found for conditions of imposed strain. The limitation of the present discussion to the initial yield behaviour of the magnesium extrusions was in recognition of these complications.

\section{CONCLUSIONS}

1) The textures developed in hot extruded commercial purity magnesium differ from those of cold extruded magnesium in having a distribution of basal poles perpendicular to the extrusion direction and parallel, or nearly so, with the transverse direction. The extrusions were flat rectangular extrusions and the textures are approximately those for plane strain deformation as in rolling. The main feature of the cold extruded texture is basal poles perpendicular to the transverse direction tilted about $15-20^{\circ}$ away from the extrusion plane normal. This fenture was present also in the hot extruded texture.

2) The cold extruded texture is accounted for by the operation of $\{10 \overline{1} 2\}$ and $\{10 \overline{1} 1\}$ twinning together with basal slip; the components of the hot extruded texture which are not present in the cold extruded texture can be explained by the operation of $\{10 \overline{1} 0\}$ or $\{10 \overline{1} 1\}$ slip.

3) The yield behaviour of the cold extruded and the hot extruded material has been investigated under tensile, compressive and plane strain deformation conditions with the largest principal stress applied in turn parallel to the extrusion, transverse and normal directions. The initial yield loci were plotted for the two materials and found to be essentially similar in form, but with some differences, attributable to the differences in texture.

4) A theoretical analysis of the anisotropy of yield stress was carried out by summing the strains over all crystal orientations in the sample, using three-dimensional crystallite distribution data and making the assumption that the strain in each crystal can be divided into three independent components : c-axis strain, plane strain perpendicular to the c-axis and shear parallel to the basal plane.
Plane strain yield loci were calculated using the results of Kelley and Hosford ${ }^{6}$ for the strength of magnesium crystals under these separate states of strain and the theoretical yield envelopes were compared with the experimental results. The agreement was remarkably good and this may be taken to indicate that an analytical approach to texture control and the use of yield anisotropy of magnesium may be rewarding.

5) There is no prospect of the plastic stress strain behaviour of magnesium, and other metals which deform by twinning, becoming amenable to analysis.

\section{ACKNOWLEDGEMENT}

We are deeply indebted to Dr. P. R. Morris and Dr. A. J. Heckler of Armco Steel Corporation, who kindly processed our texture data to produce the crystallite orientation distribution data shown in Figures 4 and 5.

\section{REFERENCES}

1. W. F. Hosford and W. A. Backofen, 9th Sagamore Conference on Material Processing, Syracuse University.

2. W. F. Hosford, A.S.M. Eng. Quart. 6, No. 4, 13-19 (1966).

3. D. J. Stratford, J. Inst. Met. 93, 87 (1968).

4. D. J. Stratford, M.Sc. Thesis (University of Aston in Birmingham, 1966).

5. B. C. Wonciewicz and W. A. Backofen, Trans. TMSAIME 239, 1422 (1967).

6. E. W. Kelley and W. F. Hosford, Trans. TMS-AIME 242, 5 (1968).

7. E. W. Kelley and W. F. Hosford, Trans. TMS-AIME 242, 654 (1968).

8. J. A. Elias and A. J. Heckler, Trans. TMS-AIME 239, 1237 (1967).

9. P. R. Morris and A. J. Heckler, Trans. TMS-AIME 245, 1877 (1969).

10. F. E. Hauser, P. R. Landon and J. E. Dorn, Trans. ASM 48,986(1956).

11. R.von Mises, Z. Angew. Math. Mech. 8, 161 (1928).

12. I. L. Dillamore and W. T. Roberts, Met. Rev. 10, 271 (1965).

13. I. L. Dillamore, E. Butler and D. Green, Met. Sci.J. 2, 161 (1968).

14. $\mathrm{H}$. $\mathrm{Hu}$, in Recovery and Recrystallisation of Metals (Interscience, New York, 1962), p. 311.

15. J. L. Walter and E. F. Koch, Acta. Met. 11, 923 (1963).

16. C. N. Reid, J. Less Common Metals 9, 105 (1965). 